\title{
SELF STABILISING UTENSIL FOR PATIENTS WITH IMPAIRED MOTOR SKILLS
}

\author{
Nikita Jain \\ Medical Electronics \\ Engineering \\ B.M.S College Of Engineering \\ Bengaluru, India
}

\author{
Rishika I.S \\ Medical Electronics \\ Engineering \\ B.M.S College Of Engineering \\ Bengaluru, India \\ Dr. Manisha Shivaram Joshi \\ Professor \\ Medical Electronics \\ Engineering \\ B.M.S College Of Engineering \\ Bengaluru, India
}

\author{
Kusuma K.B \\ Medical Electronics \\ Engineering \\ B.M.S College Of Engineering \\ Bengaluru, India
}

\begin{abstract}
Nowadays, there is a substantial increase in the number of people getting affected by Parkinson's Disease. The degradations of motor skills severely affect the daily activities of patients such as eating, writing, dressing, etc. This project basically proposes a non-invasive device that helps the patient to eat and perform day to day chores independently using the given attachments. This device consists of Arduino which directs the two stepper motor to cancel the tremors detected by the Inertial Measurement Unit (MPU6050) containing 3 axis gyroscope and 3 axis accelerometer which senses these tremors.
\end{abstract}

Keywords—parkinson, tremors, stabilising spoon, patient, gyroscope, accelerometer, Arduino, active cancellation, neuromuscular disorders, degenerative, impaired motor skills

\section{INTRODUCTION}

If you hold your hand in front of you and if you try to keep it steady you will notice something known as "tremor" which is common to happen unless you are a surgeon. These slight tremors are harmless. but there are many deadly diseases like Parkinson's and various neurological disorders where these tremors are so high that he/she is not even capable of doing daily chores such as eating, cleaning, combing which limits their independence.

They will also have deep-rooted psychological issues. As a result, people get ashamed because they fail to do simple things causing that individual to avoid all types of social gatherings etc thereby reducing the quality of life.
In this project, we are trying to help people afflicted with diseases such as Parkinson's and other neurological disorders be as independent as possible. We have come up with a selfstabilizing utensil that will counteract i.e. cancel these tremors. This device will effectively reduce unintended motions like tremors to a bare minimum.

We have used a microcontroller and servo motors to do the needful. Using these tremor compensating devices, people can execute some day-to-day tasks. With the help of our device, a person can eat independently, or use drill bits such as a screwdriver to do simple things and would not require help from others.

\section{PROBLEM ANALYSIS}

Parkinson's symptoms usually begin gradually and get worse over time. As the disease progresses, people may have difficulty walking and talking. They may also have mental and behavioral changes, sleep problems, depression, memory difficulties, and fatigue.

Both men and women can have Parkinson's disease. However, the disease affects about 50 percent more men than women. So, the chance of occurrence of this disease is more likely for mens compared to womens.

One clear risk factor for Parkinson's is age. Although most people with Parkinson's first develop the disease at about age 60 , about 5 to 10 percent of people with Parkinson's have "early-onset" disease, which begins before the age of 50 . Early-onset forms of Parkinson's are often, but not always, inherited, and some forms have been linked to specific gene mutations. 


\section{International Journal of Engineering Applied Sciences and Technology, 2021 \\ Vol. 6, Issue 2, ISSN No. 2455-2143, Pages 128-134 \\ Published Online June 2021 in IJEAST (http://www.ijeast.com)}

For many decades, doctors couldn't treat Parkinson's disease effectively, and thought it was a terminal illness. The drugs used to treat the symptoms of tremor in the late
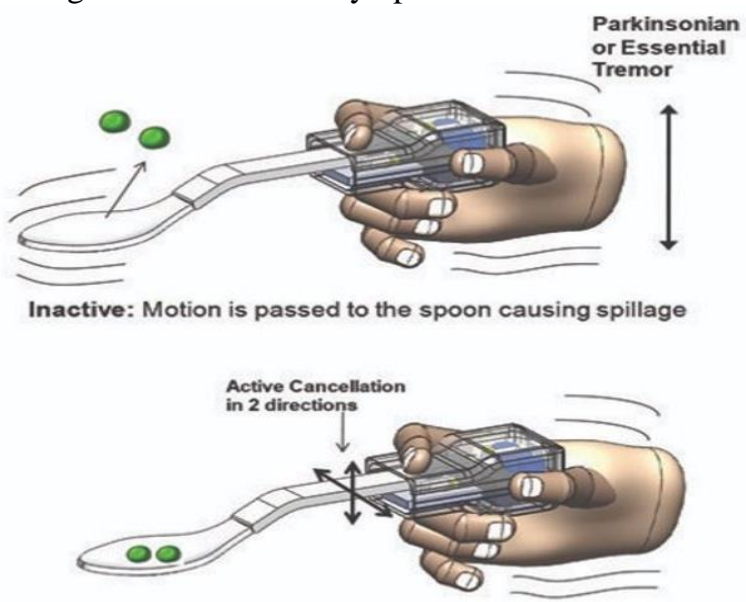

Active: Device active adjusts the utensil to oppose use tremor, providing stabilization.

Fig 1: Principle of working

1800s included arsenic, morphine, hemlock, and cannabis, according to Chrisopher Goetz's review published in September 2011 in Cold Spring Harbor Perspectives in Medicine.

Today, most Parkinson's disease patients are first treated with Sinemet, a combination of levodopa and carbidopa; the drug combination can reduce some of levodopa's side effects. These therapies are usually effective for 5 to 10 years, but eventually stop working and lead to adverse effects such as involuntary movements and tics (dyskinesia).

Though these medicines were highly used at that point of time it still contained substances which would numb the tremors but could cause major side- effects at later stages of life. People needed a solution which did not require tablets to be ingested. In other words, we noticed a dire need for a noninvasive solution to fight against such diseases.

\section{PRINCIPAL OF WORKING}

Our solution Is a Self-stabilizing Utensil/tool That Counteracts Hand tremors. This is a Non-invasive, Assistive and rechargeable Device which includes a holding bar, Rechargeable Battery, And many Attachments like

A Soup Spoon, Everyday Spoon, fork, screwdriver extensions, IMU (Inertial measurement unit) placed in The Handle Act as A Sensing Mechanism. (Active Cancellation Of Tremor Technology) It also includes FSR (force sensing resistor) which changes its resistance when a force or pressure is applied. FSR detects the Direction of Tremor And Moves Utensil In The Opposite Direction.
As the spoon is portable and quite light, It can be used in Diners, at Social events, As Well As At Home. The Rechargeable Battery Can Run approximately For 3 hours.

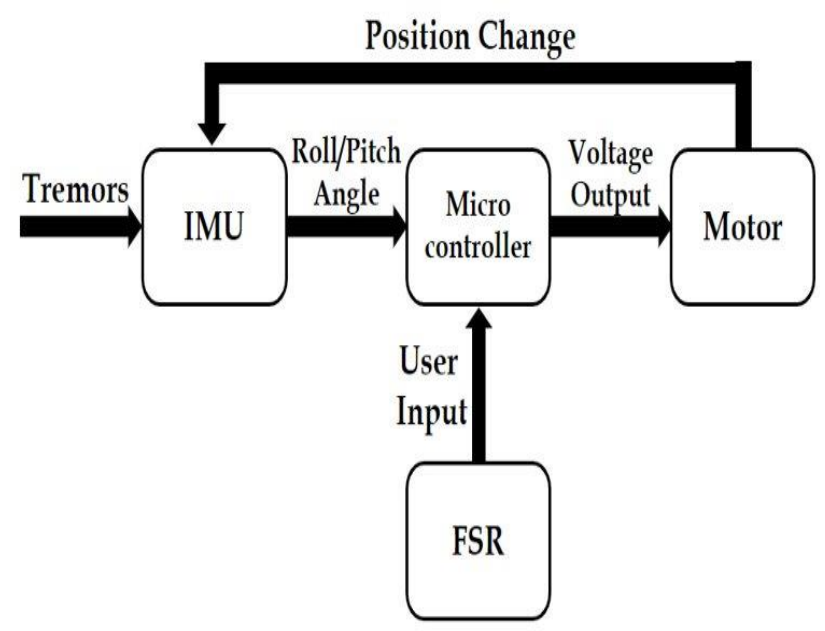

Fig 2: Block Diagram of the device

\section{METhodology}

\section{A. Components}

- Arduino Nano is a friendly board that is small, complete and breadboard with dimensions $45 \times 17 \mathrm{~mm}$ and its thickness is approximately $10 \mathrm{~mm}$ with pins attached. This breadboard is based on ATmega 328 microchip and it is equipped with fourteen digital pins and eight analog pins also among that six are PWM enabled.

Programming this Arduino board is done with the help of Arduino IDE where a mini-USB port is used for programming purposes as well as a power source. It supports both types of communications, that is I2C and SPI communication.

- MPU-6050 is the IMU unit used in our device for Motion Tracking. This combines both 3axis gyroscope and 3- axis accelerometer on a silicon die and with an onboard Digital Motion Processor which processes complex 6- axis motion fusion algorithms.

It has an auxiliary I2C master bus which gives access to external magnetometers or some sensors. MPU6050 has analog to digital converters which are 16 - bit and three in number for digitizing both the outputs of the gyroscope and accelerometer. It enables tracking of fast and slow motions precisely by allowing user-programmable gyroscopes and accelerometers. 


\section{International Journal of Engineering Applied Sciences and Technology, 2021 \\ Vol. 6, Issue 2, ISSN No. 2455-2143, Pages 128-134 \\ Published Online June 2021 in IJEAST (http://www.ijeast.com)}

- $\quad$ Micro 9g servo FS90 is the servo motor used in our device to balance and stabilize as it is one of the powerful and small motors. It provides approximately $0.127 \mathrm{Nm}$ of torque as output and can rotate about 180 degrees. This motor has only three pins where one pin is for input voltage, another pin for signals, and the last one for electric ground.

The operating voltage range for this motor is $4.8 \mathrm{~V}-6 \mathrm{~V}$, so it gives a good torque output of $1.5 \mathrm{Kg} \mathrm{cm}$ as higher torque can be achieved if the voltage is higher, so it is chosen to be used in a variety of projects. Since this servo motor has a universal S-type connector, it fits almost all kinds of receivers.

- $\quad$ A 9V battery for power supply, some connecting wires, and a breadboard.

\section{HARDWARE IMPLEMENTATION}

The $9 \mathrm{~V}$ battery supplies the operating voltage to the device, so the voltage, which is Vcc pin of Arduino, MPU6050 and Servo motor are connected to the positive terminal of the battery. Similarly the ground, which is the GND pin of Arduino, MPU6050 and Servo motor are connected to the negative terminal of the battery.

Table 1. shows the connections between the Arduino Nano microcontroller and the IMU unit that is MPU6050. Table 2. shows the connections between Arduino Nano and the two servo motors.

\section{A. Working of Device}

During the normal situation, stabilising a spoon will work like any other ordinary spoon and won't interfere in day to day chores but the moment the FSR detects force or

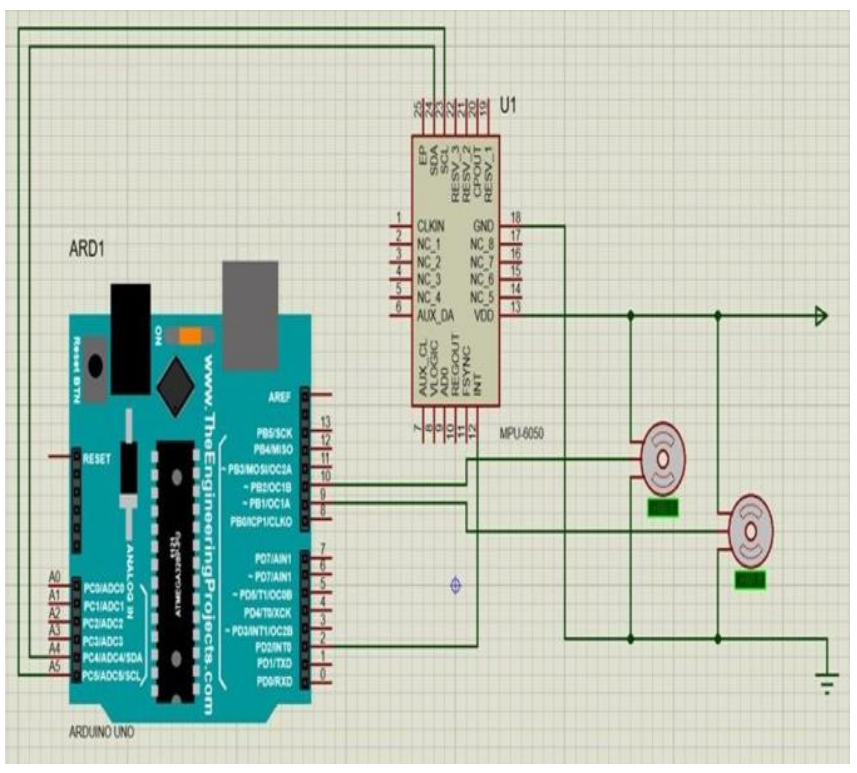

Fig 3: Circuit diagram
TABLE 1. CONNECTION BETWEEN ARduINO NANO AND MPU6050

\begin{tabular}{|c|c|c|c|}
\hline \multicolumn{2}{|c|}{ Arduino } & \multicolumn{2}{c|}{ MPU6050 } \\
\hline Pins & Function & Pins & Function \\
\hline A4 & $\begin{array}{c}\text { Serial Data line that } \\
\text { supports I2C } \\
\text { communication }\end{array}$ & SDA & $\begin{array}{c}\text { Master Bus supplies the } \\
\text { timing signal }\end{array}$ \\
\hline A5 & $\begin{array}{c}\text { Serial Clock line that } \\
\text { supports I2C protocol }\end{array}$ & SCL & $\begin{array}{c}\text { I2C Data pin where this } \\
\text { line receives as well as } \\
\text { transmits }\end{array}$ \\
\hline Pin 2 & $\begin{array}{c}\text { External Interrupt pin } \\
\text { which can configured to } \\
\text { trigger an interrupt }\end{array}$ & INT & $\begin{array}{c}\text { The Interrupt Output } \\
\text { which can be } \\
\text { programmed to raise } \\
\text { interrupt }\end{array}$ \\
\hline
\end{tabular}

pressure it sends a signal to activate IMU (MPU6050) which determines the magnitude of angle and whether it is Roll or Pitch angle.

Then the readings will go to the Arduino Nano console where input and output pins are connected which will in turn send directions to the motor to rotate according to the phase of angle. The spoon cannot cancel the full effect of tremor but it can reduce the effect by $70 \%$ which is quite helpful for the patient.

fig (5) demonstrates the 3-D model of our device in which we can see that all the mechanical parts are combined and encapsulated in a cylindrical container to make the device compact. So, when observed from left to right first is

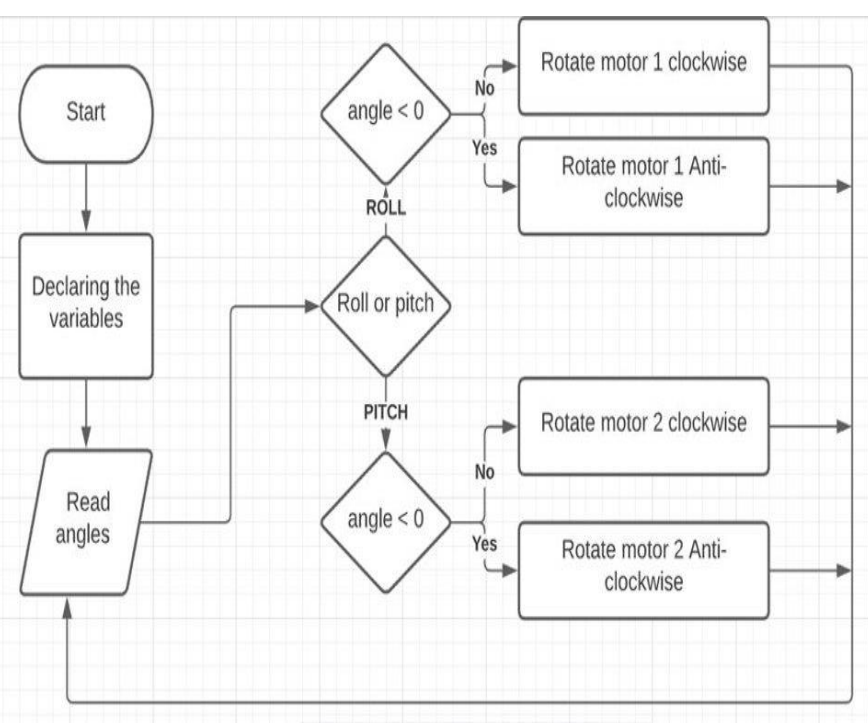

Fig 4: Flowchart of code 
TABLE 2. CONNECTION BETWEEN ARDUINO NANO AND SERVO MOTOR

\begin{tabular}{|c|c|c|c|}
\hline \multicolumn{2}{|c|}{ Arduino } & \multicolumn{2}{c|}{ Servo Motor } \\
\hline Pins & Function & Pins/Wires & Function \\
\hline Pin 9 & $\begin{array}{c}\text { Digital I/ } \\
\text { O Pin. }\end{array}$ & Signal & $\begin{array}{c}\text { Signal is given through } \\
\text { this to drive the motor 1 }\end{array}$ \\
\hline Pin 10 & $\begin{array}{c}\text { Digital I/ } \\
\text { O Pin }\end{array}$ & Signal & $\begin{array}{c}\text { Signal is given through } \\
\text { this to drive the motor 2 }\end{array}$ \\
\hline
\end{tabular}

MPU6050 which is connected to the Arduino Nano towards the right. At the end is the $9 \mathrm{~V}$ battery connected which supplies power to our device. One motor is towards the left of MPU6050, counteracts one direction and another motor which is visible outside the device counteracts the direction perpendicular to the first one.

In fig (6) 3D models of attachments are shown. These Attachments are detachable. It contains a soup spoon, fork, pen and screwdriver etc. In other words, all the handheld devices.

\section{SOFTWARE IMPLEMENTATION}

figure (4) shows flowchart of code which we used for arduino interfacing. So initially we declared the variables and read angles (detected by IMU in hardware) and applied an if condition to determine whether the tremor angle is a roll or pitch. Depending on the angle whether it is positive or negative the Arduino microcontroller directs the motor in respective directions (clockwise/anticlockwise)

The spoon is intended to have two degrees of freedom, rotational movement around the $\mathrm{x}$-axis, roll, and rotational movement around the $y$-axis, pitch. The z-axis which is orthogonal both to the $\mathrm{x}$ - and $\mathrm{y}$-axis, will have no motor for counteracting motion since two degrees of freedom will be satisfying enough for this project.

If the angle is Roll (horizontal direction) motor 1 will rotate whereas if the angle is pitch (vertical direction) motor 2 will rotate to cancel the effect of the tremors.

\section{RESUlT AND OBSERVATION}

In the (fig 7), observe the two servo motors' position is stationary. The IMU will read the tremors and Arduino will counteract these tremors by changing the position of motors.

In the (fig 8), the motor position is changed, and notice the reading before and after which is displayed below

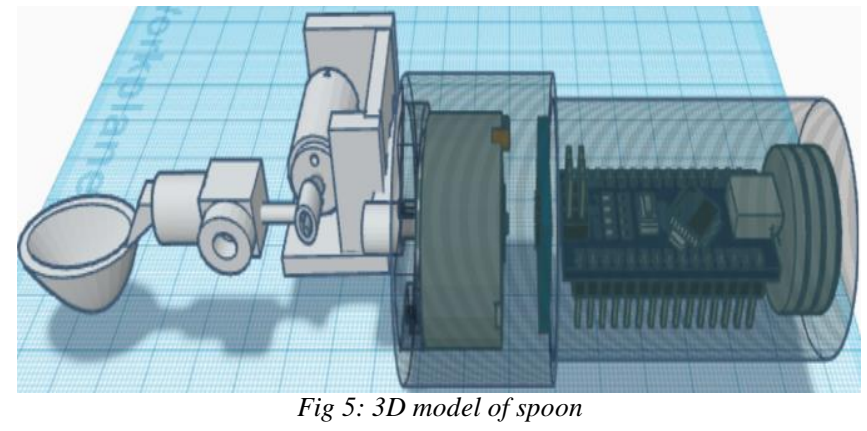

the motor. The two motors counteract each direction movement i.e motor 1 counteracts Y-direction tremors and motor 2 counteracts $\mathrm{X}$-direction tremors. There by it restores balance.

\section{FUTURE SCOPE}

We believe we have room for improvement and that would be to design a more sensitive and one-time-calibrated device with minimum intervention by medical specialists. Our model is a very specific approach for tackling the problem. We can have a more generalized approach to tackle the problem addressed, to name a few we can have self stabilizing gloves where one can perform all the action with his/her hands. We can also have a self-stabilizing pen, vest, or walking stick. This will be a ray of hope for people suffering from Parkinson's and other similar diseases. All of these use the same technology, with an extension from the design.

\section{A. Improvements in the existing device}

Also an IMU occasionally presents values that are incoherent and vary unreasonably to one another. To get proper values, i.e. clear values, a filter is needed. A complementary filter consists of one low pass filter and one high pass filter. We can also use high speed servo motors for better accuracy.

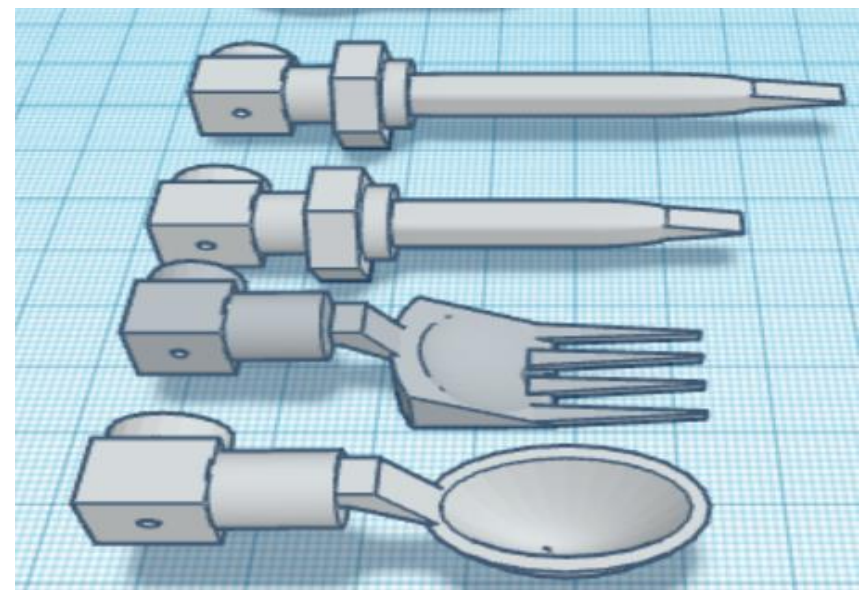

Fig 6: 3D model of attachments 


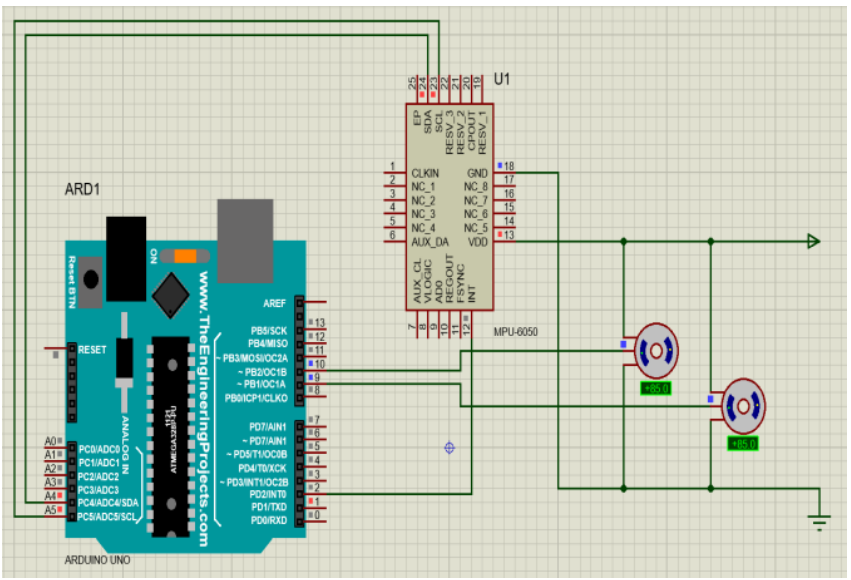

Fig 7 : Simulation output before MPU6050 readings

\section{B. Self stabilizing gloves}

The glove uses a gyroscope and accelerometer. It uses the spinning top gyroscopes which will maintain upright via spinning. These discs are made to spin fast and are coupled by hand. This device can be used to cancel the tremors and it will make the hand seem as if it is moving through a viscous treacle by resisting tremors and allowing other hand movements. These gloves use intelligent electronics to track the progress of the disease, displaying the information on a smartphone.

It is the simplicity of the device. The Glove is easy to use, reliable and compact. As wearable technology, it bridges the gap between medical treatment, which suffers from side effects, and deep brain stimulation, an invasive and exceedingly costly type of brain surgery.

\section{Self stabilizing pen}

A Korean designer with a history for design that cares about others, came up with a pen that can help people who suffer from Micrographia, Parkinson's and Neuromuscular disorders. Using a vibration motor, Gyroscope, the pen will try to correct the handwriting by countering the hand's tremors. Even better, a small computer inside the pen learns the writer's behavior and adapts to it, making it a custom tool for each person.

\section{Self stabilizing vest}

This vest is quite a modern version of the spoon as it is used in cancelling the effect of tremor as well as analysing the patterns of the tremors occurring. It is connected wirelessly to the patient's mobile and sends updates regularly.

The deep pressure therapy supplied from the weighted vest provides proprioceptive input to the joint receptors, which in turn send messages to the brain about the body's position. Proprioceptive input allows us to know, without looking at our arm,

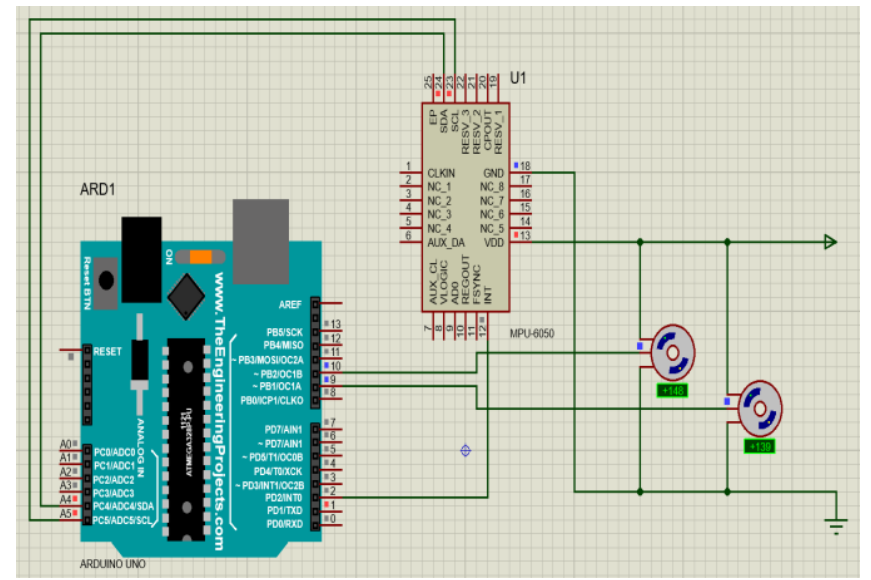

Fig 8: Simulation output after MPU6050 readings

For example, that our arm is behind us, next to our body, or up in the air. Because deep pressure helps provide proprioceptive input, weighted vests with weights correctly and strategically positioned can, therefore, help improve coordination through the increased awareness of body positioning. This sense of body position, which includes movements of the muscles, tendons, and joints, is called kinesthesia

\section{E. Walking stick}

Parkinson's Patients Walking Stick - Chalte Raho is a walking stick that stimulates movement in patients with Freezing of gait through a visual trigger in the form of a beam of light projected on the ground in front of them. It is Launched in 2016. The patient switches on the stick before he/she begins walking. As soon as the patient experiences freezing he/ she presses the button under the handle.

A laser line projected on the floor provides the patient with an external cue. He/she steps over the line and continues walking.

\section{AdVANTAGES AND Disadvantages}

\section{A. Advantages}

Self Stabilizing utensils feature ease of use for any meal as they are simple to use. There are detachable and attachment options like attachment of fork, attachment of soup spoon, attachment of regular spoon, other useful attachments like pen, screwdriver, paint, and many in place of spoon.

The optional attachments are really useful while performing everyday chores. This utensil is user friendly as it can be easily carried wherever we travel like it easily fits in a pouch so we can carry it everywhere. Since it is portable and easy to carry around, users are free to go out and need not worry about awkwardness or embarrassment.

Our device can also last long as it has a full battery for continuous power supply and lasts while performing 


\section{International Journal of Engineering Applied Sciences and Technology, 2021 \\ Vol. 6, Issue 2, ISSN No. 2455-2143, Pages 128-134 \\ Published Online June 2021 in IJEAST (http://www.ijeast.com)}

many tasks. So, users can use them happily without worrying about its longevity and finish their work and tasks with ease.

Users will have a social boost as they used to feel seclusion and social anxiety as their impaired motor skills, tremors used to make them unable to do their basic tasks. Hence they will have both a moral and social boost and become more socially active.

Our solution can make them socially active and prevent them from being isolated, so we believe this technology helps to mitigate their isolation. Since our solution helps them with their everyday chores they need not worry about awkwardness.

The designed utensil model is easy to handle as well as safe. This designed model has pretty tough resistance for breakage, flexible to use. Also it's easily washable and no worries about leakage of water on components as the design takes care of these. The detachable technique is simple and can be done whenever required.

\section{B. Disadvantages}

Some of the users who are under continuous monitoring like patients who have pacemaker or other medical electronic device implants need to use this utensil with care. Since, the self stabilizing utensil has small magnets which have magnetic strength similar to the magnetic strength of ordinary household magnets. These magnetic strength may influence the working of implants so its better to hold the utensil far as possible.

The Self stabilizing utensil is designed to handle moderate essential tremors. If the user experiences severe tremors then the utensil's performance in stabilizing may not be that effective. In case of severe tremors, recognizing them might be not so efficient. Thus balancing or stabilizing effects may not be so influential and users might experience the effects of tremors on objects held in hand.

\section{APPLICATIONS}

Self-Stabilizing utensil are mainly used by people suffering from impaired motor skills and also patients suffering from hand tremors due to Parkinson's disease. The optional attachments offered, helps to use different attachments during different situations and it follows an easy detaching technique.

Users can use this utensil while eating, as an effect of hand tremors leads to spilling of food. So this can be avoided, and users can peacefully enjoy their food without worrying about sillage. A regular spoon as well as soup spoon are available as attachments. So while having soups, a soup spoon can be easily attached and used, similarly while eating a normal spoon can be used.
Users can also make use of the fork attachment while eating, like while having fruits, salads, noodles, etc. They can easily attach the fork and use it whenever required.

Users can make use of the self-stabilizing utensil even while writing. The pen attachment feature extends the application to writing, drawing, etc. Users can also write or draw or do tasks using this pen attachment.

Another attachment extension is screwdriver, users can also make use of this attachment while doing tasks using drill bits.

\section{CONCLUSION}

The self-stabilizing utensil as observed from the previous section gave good performance thus it is satisfying the needs. It recognizes essential tremors and counteracts to stabilize the tremor action.

The device was designed with a cylindrical handle that fits most adult people's hands and it is also lightweight and easy to handle. The attachment options offered also add to advantages as they are simple to detach and use as well as helpful to perform basic tasks, like while eating they can use it as a spoon, fork, or while writing they can use it as a pen and in similar situations it is helpful for them.

This concludes that The Self-Stabilizing Utensil can be utilized by people with impaired motor skills, to a certain extent, which contributes for betterment of their life.

\section{ACKNOWLEDGMENT}

We thank and express our gratitude to our guide "Dr. Manisha Shivaram Joshi" ma'am for the guidance and support in completing the project. We would also like to extend our gratitude to HOD ma'am "Dr. Vijayalakshmi K" who provided us with the opportunity to do this project.

We would also like to thank "Dr. M.S. Suma" ma'am for smooth conduction of the course. We are deeply grateful to all the staff members of the Medical electronics Department, BMS College of engineering for supporting us in all aspects.

\section{REFERENCES}

[1] Adhavi Shri. A.S, S.Praveen Kumar and T.Aravind "Design of Mems Gyroscope to Detect and Monitor Parkinson's disease" 2013 International Conference on Smart Structures \& Systems (JCSSS-20 13), March 28 29, 2013, Chennai, INDIA

[2] H. Uchitomil, K. Suzukil, T. Nishil, M. J. Hove, Y. Wada, S. Orimo, Y. Miyakel "Interpersonal Synchronybased Dynamic Stabilization" Of the Gait Rhythm between Human and Virtual RobotClinical Application to Festinating Gait of Parkinson's Disease Patient Tokyo Institute of Technology, Midori, Yokohama 226-8502, Japan.

[3] Suprio Bhattacharya, Rahul Krishna, Brent Clay, and Christopher "Moore "sHaKe n SCALE': A 
Measurement system of Motor characteristics of Parkinson's disease" IEEE VACS 2015

[4] Politis, M., Wu, K., Molloy, S., G Bain, P., Chaudhuri, K. R., \& Piccini, P. (2010). Parkinson's disease symptoms: the patient's perspective.Movement Disorders: Official Journal of the Movement Disorder Society, 25, 16461651. doi:10.1002/mds.23135

[5] Duval, C. (2006). "The Rest and postural tremors in patients with Parkinson's disease". Brain Research Bulletin, 70 , 4448. doi:10.1016/j.brainresbull.2005.11.010

[6] Wei Wang, Xiaoyong Lv, and Feng Sun, "Design of Micromachined Vibratory Gyroscope with Two Degree-
of-Freedom DriveMode and Sense-Mode" Senior Member, IEEE, July 2012.

[7] Kavitha K. Nithya R, Shahana R K. Anju Gupta Alagappan M, "Design and Simulation of MEMS-Based Gyroscope for Vestibular Prosthesis", COMSOL Conference, 2012

[8] Wellington C. Pinheiro, Bruno E. Bittencourt, Lucas B. Luiz, Lucas A. Marcello, Vinicius F. Antonio, Paulo Henrique A. de Lira, Ricardo G. Stolf and Maria Claudia F. Castro, "Parkinson's Disease Tremor Suppression", 10th International Joint Conference on Biomedical Engineering Systems and Technologies (BIOSTEC 2017), pages 149-155, 2017. 